\title{
Calidad de vida rural y metodología cualitativa
}

\author{
Isabel Cristina Marin Arriola ${ }^{1}$ \\ Centro Universitario del Sur- \\ Universidad de Guadalajara
}

\section{Ensayo}

Material original autorizado para su primera publicación en Journal de Ciencias Sociales, Revista Académica de la Facultad de Ciencias Sociales de la Universidad de Palermo.

Recepción: 19-10-2020

Aceptación: 09-03-2021

Resumen: La calidad de vida es un constructo, que durante los últimos 30 años ha sido estudiado desde diversas disciplinas y enfoques, debido a la repercusión que logra tener en la política pública de los países. Su medición ha sido objeto de debates sociales, económicos y políticos en el mundo, pero el interés por comprender este constructo, surge de los investigadores, agencias internacionales y agenda pública debido al alto impacto que la calidad de vida tiene sobre las personas tanto a nivel individual como social. Por esta razón, el objetivo de este ensayo es exponer cómo la metodología cualitativa aplicada a los estudios de calidad de vida se convierte en una experiencia de comprensión subjetiva de la problemática social de las poblaciones de los países latinoamericanos. Además, se destacan tres contribuciones académicas que los estudios cualitativos en calidad de vida han aportado a este concepto durante el nuevo el nuevo milenio: (1) la propuesta de trabajar con las potencialidades y no con las carencias de las personas, (2) integrar a la evaluación tres áreas elementales como lo son: el político, el económico y el sociocultural, y (3) pensar la calidad de vida en grupo más que en lo individual.

Palabras clave: metodología cualitativa; calidad de vida rural; Latinoamérica; grupos sociales.

\section{Rural quality of life and qualitative methodology}

Abstract: Quality of life is a construct, that has been studied from various disciplines and approaches over the last 30 years, due to impact it has on public policy in countries. Its

\footnotetext{
${ }^{1}$ Licenciada en Nutrición por la Universidad de San Carlos de Guatemala. Magister en Gerencia de la Seguridad Alimentaria y Nutricional por la Universidad Nacional de Costa Rica. Estudiante del programa de Doctorado en Psicología con Orientación en Calidad de Vida y Salud, en el Centro Universitario del Sur, de la Universidad Guadalajara, y docente de la misma universidad.

Correo electrónico: cristinam@cusur.udg.mx
} 
measure has been the object of social, economic and political debates in the world, but the interest in understanding this construct arises from researchers, international agencies and public agenda due to high impact quality of life has on people both at the individual as well as social. For this reason, the objective of this essay is to present how the qualitative methodology applied to quality of life studies becomes an experience of subjective understanding of the social problems of populations of Latin American countries. In addition, three academic contributions standout that qualitative studies on quality of life have made to this concept during the new millennium: (1) the proposal to work with potentialities and not with shortcomings of people's, (2) integrate three into the evaluation elementary areas such as: the political, the economic and the sociocultural, and (3) think about the quality of life in groups rather than individually.

Keywords: qualitative methodology; rural quality of life; Latin America; social groups.

\section{Introducción}

Para el ser humano, la concepción de una vida feliz se presenta desde tiempos antiguos a través de las disertaciones de diversos filósofos griegos. Para Aristóteles (2007), la Eudaimonia se concebía como una manera de vivir que se expresaba activamente en excelencia de carácter o en virtud. Mientras que Sócrates (2006), consideraba que la perfección moral era la verdadera felicidad del ser humano. Por su parte, Platón (2000) definió la felicidad como la combinación de tres elementos: el bien, la verdad y la justicia, los cuales conformarían un estado feliz.

Jeremy Bentham (1748-1832) fue un filósofo, contemporáneo, cuya influencia es importante en los fundamentos filosóficos del concepto calidad de vida. Su principal aportación a la concepción de la felicidad estuvo basada en el pensamiento de que "el mayor bien es el de maximizar el placer para el mayor número de personas" (Sirgy, 2012, p.7), mostrando una corriente política progresista para inicios del siglo XVII. Sin embargo, derivado de lo anterior, se infiere que los orígenes filosóficos de calidad de vida están sustentados en dos pensamientos esenciales: por un lado, la concepción aristotélica de eudaimonía que se traduce como florecimiento, bienestar, éxito y oportunidad de liderar una vida con propósito y significado. Por otro lado, la concepción utilitarista de Bentham quien consideraba el bienestar humano de utilidad hedónica lo que significa: contentamiento y que se puede representar hoy en día como un estado mental de satisfacción individual (Sirgy, 2012, p. 6,7).

A lo anterior es importante agregar que, aunque esas son las bases filosóficas de la calidad de vida, otros pensadores e investigadores a través del tiempo han reforzado la idea de que la condición de felicidad es la que motiva fuertemente al ser humano a tomar acciones 
frente a la vida (Diener, et al. 2018; Lecomte, 2018; Rojas, 2014). En palabras de Sirgy (2012), esta aportación integra en gran medida la parte psicológica del concepto de felicidad, que es el antecedente más cercano del concepto de calidad de vida.

Algunos investigadores coinciden en señalar que a partir de la segunda post guerra, el concepto de salud que declara la Organización Mundial para la Salud (OMS) en 1948, cambia la forma de entender la salud y por ende el bienestar y calidad de vida de las personas hacia una dimensión más amplia, incluyendo dentro de ella los aspectos físico, mental y social, para dejar atrás el concepto de salud únicamente como la ausencia de enfermedad (Alcántara-Moreno, 2008; Moreno Jiménez y Ximénez Gómez, 1996; Urzúa y Caqueo-Urízar, 2012). Es así que, en el siglo XX, durante la década de los años sesenta, empieza un interés por estudiar y perfilar un concepto sobre calidad de vida de los habitantes de los países desarrollados (Ardila, 2003; Noll, 2002). En esa misma década, la OMS define calidad de vida como "La percepción que un individuo tiene de su lugar en la existencia, en el contexto de la cultura y del sistema de valores en los que vive y en relación con sus objetivos, expectativas, normas y preocupaciones" (OMS, 1994, p.29).

Ya instituida desde la OMS la noción de calidad de vida, inicia su aparición en la agenda de los gobiernos occidentales como por ejemplo en el de John F. Kennedy y Lyndon B. Johnson en los Estados Unidos (Land y Michalos, 2018). Simultáneamente, la concepción de calidad de vida se integró en la economía del siglo XX a través del sistema de consumo de bienes y la obtención de comodidades para el bienestar, lo que al paso de los años se llamó economía del bienestar. La economía del bienestar a su vez, tuvo sus bases en sociedades urbanas desarrolladas y con pensamiento liberal; como consecuencia, las primeras investigaciones sobre calidad de vida se basaron mayormente en datos socioeconómicos (Urzúa y Caqueo-Urizar, 2012). No es sino hasta finales de la década de los años sesenta, que la calidad de vida se empieza a estudiar desde una perspectiva subjetiva de salud, donde se incorporaron elementos psicosociales que afianzaron la conceptualización teórica de la calidad de vida desde la psicología (Casas, 1999). Al inicio del nuevo milenio los aportes de la investigación cualitativa en calidad de vida proporcionaron un conocimiento que complementó y dinamizó a los estudios cuantitativos (Calderón, 2002). También, la investigación cualitativa al integrar la visión sociológica y antropológica de estudiar los asuntos que conforman la vida cotidiana, permitió conocer las creencias, los valores, las actitudes, las percepciones, las opiniones y hasta las formas de actuar de las personas respecto a su calidad de vida (Bedregal et al, 2017; Tonon, 2015; Villegas y González, 2011). Esto ha servido para tomar decisiones a nivel individual y colectivo sobre salud, educación, economía, y desarrollo con una visión diferente: la visión de los individuos. Tomando en consideración estos antecedentes, el objetivo del presente ensayo es mostrar cómo la metodología cualitativa aplicada a los estudios de calidad de vida, se convierte en 
un proceso de comprensión subjetiva de la problemática social de las poblaciones de los países latinoamericanos.

\section{Argumentación}

Durante los años setenta en Europa se inician los debates de tipo filosófico sobre la calidad humana. Peccei (1976) propone visualizar la calidad humana a nivel de todas las personas en el planeta, esta nueva visualización del concepto permitió trasladar el concepto de calidad de vida de un valor individual al concepto de aspiraciones colectiva, lo cual desencadenó el uso del concepto en el desarrollo de políticas públicas, además de múltiples y variadas intervenciones profesionales. En el campo de la política y de la intervención social se relacionó de inmediato con la prestación de servicios y la satisfacción de los usuarios (Casas, 1999). Los estudios realizados por Andrews y Withey (1976) y en el de Campbell et al. (1976) se fundamenta el concepto de calidad de vida desde la concepción psicosocial, que a su vez permite concebir el bienestar social como parte de la calidad de vida de las personas.

Unos años más tarde se puso en apogeo el concepto de desarrollo humano, y algunos gobiernos lo integraron como la parte subjetiva del propósito de las políticas públicas de sus países. Derivado de esto surgen teorías sobre cómo medir el desarrollo humano desde una perspectiva más integral que informase sobre la situación social a nivel de países o regiones. Esta importante contribución fue hecha por el Programa de las Naciones Unidas para el Desarrollo (PNUD) y posteriormente profundizada por investigadores como Coleman (1988) y Putnam, et al. (1993) quedando relacionado el desarrollo con la calidad de vida de las personas y no con los objetos.

También se reconoce las contribuciones teóricas de filósofos, economistas, políticos y sociólogos como: Abraham Maslow (1943), Manfred Max-Neef (1994), Amarthya Sen (comentado por Hernández,1998), que concibieron la evaluación de la calidad de vida desde las necesidades básicas y capacidades de las personas con una visión integral de las condiciones de vida.

Con relación a organismos internacionales, en 1990, las Naciones Unidas a través del Programa para el Desarrollo (PNUD, 1990) establece el índice de desarrollo humano (IDH) como indicador para medir la calidad de vida. Este indicador clasifica a los países a partir del índice de alfabetización, tasas de natalidad, esperanza de vida, y otros indicadores diferentes a los utilizados macroeconómicamente antes de esa fecha. Existen otros índices que también han sido protagónicos en medir las condiciones de vida de las personas como: The Canadian Index of Well-being, The Gallup Health ways Well-being Index, The Social Progress Index y 
The Child Well Index, todos mencionados y estudiados por Land y Michaelos (2018) y señalados por estos autores como bases de la evaluación objetiva de calidad de vida.

Posterior a esto, en el siglo XXI la calidad de vida está íntimamente ligada al contexto y a la experiencia de cada persona, es decir, a su propia vida social y a su propia percepción de lo que le provoca satisfacción y felicidad. Así mismo, en los últimos años se han integrado temáticas relacionadas a medio ambiente, geografía humana y la ecología (Silva et al. 2012).

El término calidad de vida se entiende más como un constructo que como un concepto, ya que cumple con ser una entidad hipotética de algo que se sabe que existe pero que es de difícil definición (González et al. 2017). Actualmente, no existe consenso acerca de su definición. Algunos autores señalan que la calidad de vida es un constructo global e inclusivo en el que durante los últimos años intervienen distintas profesiones y disciplinas, lo cual puede explicar la falta de consenso (Ardila, 2003; Carpio et al. 2000; Moreno Jiménez y Ximénez Gómez, 1996; Urzúa y Caqueo-Urízar, 2012). La tabla número 1 muestra un resumen cronológico de definiciones sobre calidad de vida, citadas en diferentes fuentes, donde se observa la tendencia a concebirla desde un tipo de indicador: objetivo, subjetivo o mixto/ integrador.

Tabla 1

Conceptos de calidad de vida

\begin{tabular}{|c|c|c|c|}
\hline $\begin{array}{l}\text { Indicador } \\
\text { de } \\
\text { tendencia }\end{array}$ & Definición & $\begin{array}{l}\text { Cronología } \\
\text { disciplinar }\end{array}$ & Disciplina \\
\hline \multirow{6}{*}{ Subjetivo } & $\begin{array}{l}\text { Hace referencia a `bienestar’, ‘felicidad`y } \\
\text { `satisfacción con la vida. }\end{array}$ & $\begin{array}{l}\text { Bradburn en } \\
1969\end{array}$ & Psicología \\
\hline & $\begin{array}{l}\text { Sentimiento personal de bienestar, de } \\
\text { satisfacción/insatisfacción con la vida o de }\end{array}$ & $\begin{array}{l}\text { Dalkey y } \\
\text { Rourke, } 1973\end{array}$ & \multirow{5}{*}{ Medicina } \\
\hline & felicidad/infelicidad. & \multirow{2}{*}{$\begin{array}{l}\text { Viney y } \\
\text { Westbrook en } \\
1981\end{array}$} & \\
\hline & $\begin{array}{l}\text { Señala el carácter multidimensional y } \\
\text { complejo del concepto calidad de vida y lo } \\
\text { refieren a la manera en que los pacientes } \\
\text { hacen significativas sus experiencias. }\end{array}$ & & \\
\hline & $\begin{array}{l}\text { Se hace referencia a una serie de tópicos, } \\
\text { como quejas físicas y psíquicas, } \\
\text { sentimientos de bienestar y funcionamiento } \\
\text { sexual y actividades cotidianas. }\end{array}$ & $\begin{array}{l}\text { Van Dam en } \\
1986\end{array}$ & \\
\hline & $\begin{array}{l}\text { Es la apreciación que el paciente hace de } \\
\text { su vida y la satisfacción con su nivel actual } \\
\text { de funcionamiento comparado con el que } \\
\text { percibe como lo posible o ideal. }\end{array}$ & $\begin{array}{l}\text { Celia \& Tulsky } \\
\text { en } 1990\end{array}$ & \\
\hline Objetivo & $\begin{array}{l}\text { La posesión de los recursos que se } \\
\text { necesitan para la satisfacción de las } \\
\text { necesidades o deseos, participación en }\end{array}$ & $\begin{array}{l}\text { Shin y Johnson } \\
\text { en } 1978\end{array}$ & Economía \\
\hline
\end{tabular}




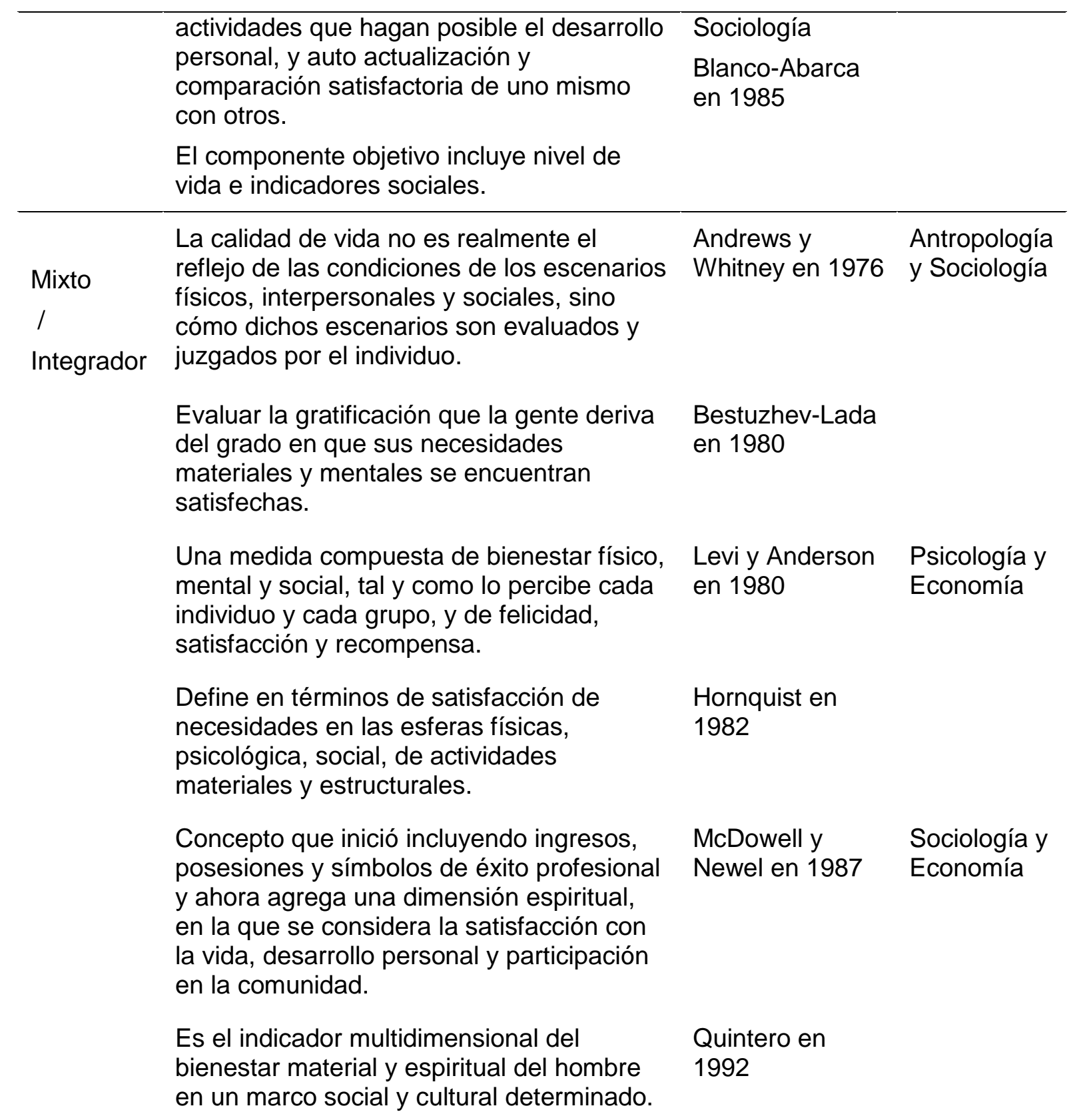

Fuente: elaboración propia a partir de Bobes et al. (1993); González et al. (1993); Urzúa y Caqueo-Urízar, (2012).

El carácter multidisciplinario de su abordaje, ha hecho que en la literatura se utilicen indiferenciadamente términos como bienestar, felicidad y satisfacción con la vida refiriéndose a calidad de vida (Casas, 2015; Fernández-López et al. 2010; Urzúa y Caqueo-Urízar, 2012). Así mismo, en el concepto están incluidos dos componentes básicos del desarrollo de la vida humana: las condiciones objetivas y las subjetivas. De acuerdo con Veenhoven (2000) la primera se refiere a la valoración que hace un individuo externo sobre los aspectos 
económicos que le permiten a la persona alcanzar la satisfacción de las necesidades vitales como: comida, casa, trabajo, etc., mientras que la condición subjetiva hace referencia al bienestar, felicidad o satisfacción con la vida autoevaluada por la misma persona. Tonon (2003) complementa al respecto y señala “... que las condiciones objetivas inician el camino hacia el bienestar subjetivo, y que las condiciones humanas subjetivas son tanto causa como efecto de las objetivas" (p. 32).

Hoy en día, los enfoques con que se estudia el constructo también contribuyen a que aparezcan diferentes concepciones. Por ejemplo, el enfoque cuantitativo tiene como objetivo medir o analizar la calidad de vida a través de la cuantificación o medición, mientras que el enfoque cualitativo tiene como propósito escuchar a las personas al relatar sus experiencias, desafíos y problemas para comprender su calidad de vida (Dennis et al. 1993).

Este ensayo se centra en comprender las ventajas y espacios de oportunidad que provee la aplicación de los métodos de investigación cualitativa a los estudios de calidad de vida en grupos de población. Señala Izcarra (2014) que los métodos cualitativos pueden ser simples y concretos en su forma, ya que se refieren a acercamientos metodológicos que no son cuantitativos, por ejemplo, la ausencia de una muestra preconcebida, comprender al "sujeto" y no al "objeto" en estudio. No obstante, para Vieytes (2009) la metodología cualitativa es compleja, ya que se basa en el razonamiento inductivo, que lleva al investigador a partir de la realidad concreta y de datos específicos a teorizar, es decir, ir de lo particular a lo general. Esto con la finalidad de comprender la realidad social, lo que la gente vive y cómo lo vive. Esta complejidad se ve reflejada en el aporte de Tonon (2015), quién los refiere como aquellos métodos que estudian significados intersubjetivos, situados y construidos, profundizando en la comprensión de la vida social tal como ésta se desarrolla y explorando el significado para sus actores.

Es importante mencionar que distintos investigadores (Lichtman, 2013; Lyn y Morse, 2012; Punch y Oancea, 2014), coinciden en que este tipo de estudios se utilizan para analizar la forma en que los individuos perciben y experimentan fenómenos que los rodean. Metodológicamente, se caracteriza por no buscar probar, medir, ni explicar el fenómeno, sino comprender los fenómenos sociales desde las experiencias y puntos de vista de los actores sociales, y el entendimiento de los significados que éstos asignan a sus acciones, creencias y valores, como lo mencionan Iñiguez (2004) y Wynn y Money (2009). Para Iñiguez (2004), esto significa dar cuenta de la realidad social, generar nuevas ideas o teorías y, depender en menor grado de conceptos preexistentes.

Otras características importantes del proceso de la investigación cualitativa son: 1) un diseño iterativo, que hace que la ruta no sea lineal, sino recurrente; 2) un planteamiento de actividades sucesivas y organizadas, es decir, pasos y pruebas a desarrollar, así como 
técnicas para reconstruir categorías que emplean los participantes en la conceptualización, recolección de datos; y 3) métodos de análisis, un proceso de abstracción en las unidades de análisis que se revelan en el transcurso de la observación y descripción. (Iñiguez, 2004; Monje, 2011; Quecedo y Castaño, 2002).

En la investigación cualitativa, la selección de la muestra es iterativa y se va integrando en diferentes momentos del proceso. Por ejemplo, Flick (2012) menciona que en un estudio se toman decisiones sobre: personas a entrevistar, proveniencia de los grupos, y qué entrevistas se deben profundizar para luego seleccionar el texto. Esto indica que el muestreo se va construyendo con el avance de la investigación, que será el inicio del hilo conductor que llevará al desarrollo total del estudio.

Los instrumentos se eligen de acuerdo al fenómeno estudiado, a los objetivos que se persiguen, al contexto donde se desarrolla la investigación, al colectivo con el que se trabaja y a cualquier limitación que pueda enfrentar el investigador (Ibáñez e Iñiguez, 1996, Izcarra, 2014). Metodológicamente, es importante mencionar que en la investigación cualitativa los instrumentos auxiliares no son estandarizados, y esto permite trabajar con múltiples fuentes de datos simultáneamente (Lichtman, 2013). Existen dos tipos de datos, (1) los datos primarios, que son aquellos que se recolectan de primera mano a través de las técnicas como las entrevistas (se obtienen las prácticas discursivas) o las observaciones (directas o indirectas que permiten recolectar lo que se hace), y (2) los datos secundarios son todos aquellos datos que ya han sido producidos y procesados por otros, es decir, todos aquellos documentos escritos, documentales, programas de radio o censos, pero que sirven de campo.

Las técnicas de investigación cualitativa más utilizadas son: la observación, las notas de campo y las entrevistas. La observación tiene como propósito explorar y describir ambientes, comunidades, subculturas y los aspectos de la vida social, analizando los significados y a los actores que la generan (Patton, 1990). También sirve para comprender procesos, vinculaciones entre personas y sus situaciones, experiencias o circunstancias e identificar problemas sociales, hasta generar hipótesis para futuros estudios (Daymon y Hollowey, 2010; Grinnell, 1997; Patton, 1990).

Tonon (2015) identifica dos tipos de observaciones que dan como resultado descripciones del fenómeno: la descripción fina y la descripción densa. La primera permite describir sin llegar a mostrar las intenciones, motivos, significados o circunstancias. Y en la observación densa se dedica a descubrir el conocimiento subyacente, las relaciones estructurales de las personas bajo estudio y, por lo tanto, permiten encontrar detalles, contextos, emociones y afiliación a las redes y al poder. 
Existe también la observación participante, la cual implica el dominio y aplicación de varias técnicas en un momento dado. Requiere que el investigador se integre en el campo como un miembro más, donde juega uno o varios roles participantes, y utiliza una lógica y un proceso de indagación abierto, flexible, oportunista que lo lleva a redefinir el problema y a utilizar otros métodos para reunir información y lograr teorizarlo (Jorgensen, 1989).

Las notas de campo se consideran el testigo escrito de algo que sucedió durante la investigación, que se observó y que es relevante para la investigación. Estas pueden ser de tres tipos: las metodológicas, las teóricas y las descriptivas. Las notas de campo incluyen descripciones de personas, acontecimientos y conversaciones, tanto como las acciones, sentimientos, intuiciones o hipótesis de trabajo del observador. Las notas de campo deben ser precisas con relación a la duración de los acontecimientos y las conversaciones (Taylor y Bogdan, 1987). Existen hoy en día variedad de medios de registro: computadoras personales, tableta, teléfono móvil, grabadora de voz o de video, papel y lápiz; también se incorporan en estas las fotografías, los mapas y diagramas.

Las entrevistas a profundidad, son un proceso comunicativo mediante el cual el investigador obtiene la información más densa del entrevistado (Alonso, 2003; Tonon, 2015). Menciona Álvarez-Gayou (2007) que esta técnica se caracteriza en que el entrevistador tiene un dominio sobre el tema y puede llegar a comprender el mundo desde la perspectiva del entrevistado. Por su parte, Izcarra (2014), agrega que se distinguen tres elementos básicos en este ejercicio: “i) diálogo o encuentro cara a cara, ii) carácter holístico del diálogo, y iii) la búsqueda de la comprensión de la perspectiva del entrevistado respecto de su vida, experiencias, situaciones, ideas y valores" (p.141).

Una de las premisas que diferencia al método cualitativo del cuantitativo, es que no es reproducible, no se puede repetir (Martínez Miguelez, 2006). A su vez, los instrumentos que se usan para la recolección de datos son abiertos a los cambios que puede presentar el fenómeno estudiado -diseño iterativo-. Lo flexible que es, se debe a que el mundo que se observa está en constante evolución y cambio, por eso los resultados serán diferentes para cada investigador no obstante utilice el mismo diseño, ya que su subjetividad puede ser diferente. (Izcarra, 2014). En este orden de ideas, coinciden Latiesa (1994) e Izcarra (2014) que esa condición no significa que la investigación cualitativa no tenga resultados precisos, estables, coherentes y exactos, sino que, por el contrario, es un instrumento totalmente válido y confiable.

En contraste al pensamiento positivista donde la confiabilidad está basada en un universo estático, el concepto cualitativo de confiabilidad está fundamentado en un mundo social en proceso de construcción (Baxter y Eyles, 1997; Marshall y Rossman, 1999; Seale, 2001). Por lo tanto, la confiabilidad en la investigación cualitativa se basa en la concepción 
de constante cambio del mundo social, es decir, desde una perspectiva cualitativa, es necesario comprender la confiabilidad de la investigación en el continuo proceso de construcción de la realidad social.

Entonces, si los métodos cualitativos se enfocan en comprender los fenómenos, estudiándolos desde la perspectiva de los participantes en su ambiente natural y en relación con el contexto, ¿qué forma toman cuando se aplican a los estudios sobre calidad de vida?

Cuando se trata de entender la pertinencia e importancia de utilizar métodos de investigación cualitativa para el estudio de la calidad de vida, Tonon menciona, que:

"... adopting a particular methodology implies a philosophic, theoretical and political decision and, in the case of qualitative methodology, it is an essential approach in understanding people's experiences of wellbeing and discovering new issues related to quality of life." [Adoptar una metodología específica, implica una decisión filosófica, teórica y política, y en el caso de la metodología cualitativa, se convierte en un enfoque esencial para comprender las experiencias de bienestar de las personas y descubrir nuevos temas relacionados a calidad de vida.] (Tonon, 2015, p.3).

Para comprender más a fondo la aplicación de la investigación cualitativa a la calidad de vida, es importante hacer referencia a dos investigadores que han contribuido a sustentar estas ideas. Por una parte, Casas, quién desde 1999 propuso que la calidad de vida es un concepto que se refiere a la acción conjunta de las personas para evaluar aquello que les afecta, lo cual lleva implícito un significado político y a su vez contribuye a estudiar el bienestar físico y psicológico para relacionarlo con las necesidades materiales y socioemocionales e integrar las mediciones psicológicas y sociales de la percepción de la evaluación personal.

Esta visión se complementa con Ferris (2006), quién consideró la dependencia de las personas a la estructura social, por lo que propuso que, al evaluar la calidad de vida en el colectivo, se tomaran en cuenta las condiciones demográficas, culturales, psico-sociales y las instituciones privadas y gubernamentales que son parte del contexto de una persona o un colectivo. En esa misma línea de ideas, Veenhoven (2000) fundamentó que existen condiciones exógenas y endógenas que definen la calidad de vida de las personas. Por lo tanto, desde estas perspectivas, Tonon (2015) menciona que es necesario que en los estudios sobre calidad de vida el investigador relate a "sujetos" y esto dará como resultado un proceso de investigación social e histórico. Esto es considerable, ya que humaniza la investigación, además de concientizar el hecho de coincidir en un espacio único de tiempo y espacio, donde la comprensión del otro es el mayor valor de la investigación.

Agrega Tonon (2003) que la investigación cualitativa, además, puede aportar una mirada diferente, y más cercana a la realidad que las personas desde su contexto pueden 
percibir, describir y hasta conceptualizar sobre su calidad de vida, incluyendo el contexto político y sus derechos legítimos.

En los últimos años, una contribución importante de los estudios cualitativos en calidad de vida, es que han abierto nuevas betas o mostrado nuevos escenarios para la investigación de dicho constructo desde variadas disciplinas y otras metodologías utilizadas, tal como el estudio etnográfico y, de caso; sin embargo, de acuerdo con Tonon (2015), no han sido muchos los estudios desarrollados utilizando métodos cualitativos en el campo de la calidad de vida. Por ejemplo, en el Journal of Applied Research in Quality of Life, que es la revista oficial de la International Society for Quality of Life Studies, se reportó que del 2006 al 2013 se habían publicado solamente tres estudios cualitativos sobre calidad de vida. De igual manera, reporta tres proyectos de investigación realizados desde la Facultad de Ciencias sociales de la Universidad Nacional de Lomas de Zamora, Argentina. A pesar de esta escasez, los estudios cualitativos en la temática son importantes para reforzar las nuevas ideas y considerarlos una fuente de oportunidades para los jóvenes investigadores.

Por otra parte, los estudios que han hecho Mikkelsen (2007) así como Gordziejczuk y Mikkelsen (2019) sobre calidad de vida desde la perspectiva geográfica y espacial, han permitido pensar más a fondo en lo que llaman vínculos entre sociedad y territorio, que es el contexto que rodea e influye en la calidad de vida de los grupos sociales. Por ejemplo, en la revisión sistemática de Gordziejczuk y Mikkelsen (2019) sobre la calidad de vida y el espacio geográfico estudiado en los últimos 20 años en Argentina, se resalta que, de 32 artículos analizados, únicamente dos fueron trabajados en localidades rurales. Estos estudios permitieron definir elementos esenciales de la investigación a nivel rural y descubrir que al medir los aspectos rurales con indicadores urbanos se produce un acercamiento inexacto. Además de comprender que la connotación del espacio rural se refiere a la revalorización de estos ambientes desde diferentes aspectos como su identidad, cultura y patrimonio, lo cual forma parte de la dimensión social de un grupo y por, sobre todo, considerar que hoy en día lo rural se vive en una interdependencia permanente con lo urbano, sin sustituirse uno por el otro (Mikkelsen, 2007).

Por consiguiente, las investigaciones en calidad de vida que utilizaron el método cualitativo a inicios del nuevo milenio y que fueron emprendidas en Argentina por Graciela Tonon (2003, 2005, 2008, 2009a, 2015), permitieron que el concepto de calidad de vida incursionara con una connotación psico-social, política y sociocultural definida en sólidas conceptualizaciones de autores contemporáneos como Andrews y Withey (1976), Casas (1999 y 2015), Campbell, et al. (1976) y Evans et al. (1985). Este es un avance importante que abre las puertas para considerar la calidad de vida desde contextos específicos, contemporáneos y comunitarios. 
En esta misma línea, surge lo que López-López (2015) explica como "calidad de vida rural", es decir una categoría de calidad vida, entretejida desde la mirada de la vida rural en el contexto latinoamericano y cuyos vínculos centrales son:

i. Vínculo político, derivado de las libertades de las personas que viven en lugares diferentes a lo urbano, quienes participan activamente y efectivamente en las decisiones que afectan o pueden afectar lo que se considera una buena vida rural, por sus familias y comunidades alrededor, donde están inmersos.

ii. Vínculo económico, reflejo de las oportunidades de usar los recursos físicos y materiales del medio ambiente para ejecutar acciones que ellos consideran necesarias para tener una buena vida rural.

iii. Vínculo sociocultural, derivado de las oportunidades para acceder a los derechos fundamentales que el Estado debe proveer.

Estas consideraciones tienen fundamento en el concepto tradicional de bienestar social, elaborado por autores como Andrews and Withey (1976) y Michalos (1980). Por otra parte, Toscano y Molgary (2019), mencionan que el estudio sobre la calidad de vida de las diferentes comunidades sudamericanas se ha convertido en un nuevo segmento de investigación y que permite hacer énfasis en los elementos más destacados del entorno comunitario que forman parte de la percepción de los sujetos sobre su propia calidad de vida. A estas tendencias abona Tonon (2003) su propuesta de trabajar en la resolución de los problemas de las personas a través de sus potencialidades en lugar de sus carencias.

Es importante aclarar que también existe literatura escrita desde el enfoque mixto, lo que permite un acercamiento más integral a la temática. Además, es desde la visión de los estudios sociales, que surge la importancia de dar un nuevo sentido a las investigaciones comunitarias en calidad de vida, considerando a las personas como sujeto y protagonista de su propia calidad de vida enmarcada en un contexto cultural, socio-político y psicológico. Por ejemplo, en el libro Reflexiones Latinoamericanas sobre Investigación Cualitativa (compilado por Tonon, 2009b) un grupo de investigadores hacen un aporte desde su propio contexto para presentar los pensamientos, acciones y relaciones de aquellos sujetos que plantearon su calidad de vida en un tiempo y espacio determinado en varios países latinoamericanos. También muestra la importancia del aporte de los estudios geográficos a las disciplinas sociales, a través de poner en contacto las categorías teóricas con un espacio y tiempo específico. La geografía es entonces para Lucero (2009)

“...la encargada de aportar el lenguaje espacio-temporal necesario con el fin de interrelacionar la esfera del análisis sustancial con la esfera del análisis empírico que se concreta en el espacio geográfico" (p.141). 
En contraposición, Gómez-Álvarez y Ortiz (2016) exponen en su estudio sobre el estado de bienestar subjetivo en Latinoamérica, que hoy en día, siguen surgiendo temas como pobreza, desigualdad y carencias objetivas que aumentan las estadísticas de índices económicos, políticos y sociales y que provocan malestar a la población que, a su vez, destacan la vida de resistencia en la que se ha vivido por años. Por lo que es complejo articular la visión de los indicadores del bienestar subjetivo y la calidad de vida ante una dura realidad social.

En el caso particular de México, la ruralidad pertenece a los estudios de sociología sobre el campesinado y los indígenas. La cual, de acuerdo con Lutz (2014), se abrió paso especialmente a finales del siglo XIX y continuó por el siglo XX con un impulso fuerte de dos hechos históricos mexicanos: la Revolución y el reparto de tierras. Debido a esto la sociología rural tiene fundamentos teóricos en cuestiones agrarias, raciales y de organización social en el campo "Y no fue sino hasta la década de los años setenta, que en México se discernió la sociología rural del desarrollo rural" (Lutz, 2014, p. 165). En el siglo XXI, ha habido avances en algunos indicadores sociales como educación, salud y pobreza (CONEVAL, 2017), pero en cuestión de medición de la calidad de vida o bienestar subjetivo, Arita (2016) menciona que recientemente se dejaron de medir las condiciones de la población a través de indicadores objetivos y levemente empezaron a surgir en 2006 las observaciones desde la visión de la calidad de vida.

Por lo tanto, al agregar al bienestar subjetivo la condición de grupo rural, se convierte en una propuesta nueva y fresca para comprender desde una visión multi e interdisciplinaria la calidad de vida de los grupos sociales de Latinoamérica. A esto se debe agregar la visión de Conill (1984) con relación a que Latinoamérica es una potencia dormida de escenarios geográficos que nada restan a los escenarios de Europa y que con dichos recursos naturales "debidamente acondicionados y habilitados" (Conill, 1984, p. 42) posibilitarían el desarrollo y mejoramiento de la calidad de vida de la región. Así mismo, advirtió Conill que, en caso de no atender a la ordenación territorial en Latinoamérica, a través de la integración de tecnología adecuada y gran sensibilidad social, para estos días se acelerarían crisis tales como la erradicación masiva de población, hambre y migraciones. Lo cual lleva a reflexionar sobre la importancia de encontrar mejores procedimientos para investigar y comprender la calidad de vida desde una visión integral de las personas y sus contextos, para encontrar nuevas formas de superar las actuales crisis. Las comunidades, poblaciones o colectivos no son pasivos, sino por el contrario, están en constante actividad para resolver sus problemas de manera individual y colectiva. Por lo tanto, como investigadores, un cometido es conocer de las comunidades lo que quieren, en lo que creen, qué aceptan y qué no, para iniciar a forjar un puente de conocimiento que permita la ayuda mutua (Gómez-Álvarez y Ortiz, 2016). 


\section{Conclusión}

El presente ensayo permite evidenciar que la metodología cualitativa es una herramienta indispensable e integrativa cuando se profundiza en conocimientos sobre la calidad de vida. Permitió, también, aclarar que la calidad de vida es todo aquello que las personas perciben, aspiran y/o necesitan para ser felices, para satisfacer sus demandas en la vida, para vivir bien en un territorio determinado bajo el ejercicio de sus derechos. Por otra parte, se muestra que es acertada la propuesta de la academia latinoamericana sobre la calidad de vida coincidiendo en los ejes político, económico, y sociocultural para poblaciones especialmente las rurales. Resta al futuro, profundizar como países o preferente como regiones en los estudios de calidad de vida para resolver de mejor manera las actuales crisis.

\section{Referencias bibliográficas}

Alcántara-Moreno, G. (2008). La definición de salud de la Organización Mundial de la Salud y la interdisciplinariedad. Sapiens. Revista Universitaria de Investigación, 9(1), 93107. https://www.redalyc.org/pdf/410/41011135004.pdf

Alonso, J. C. (2003). El estudio de caso simple: Un diseño de investigación cualitativa. Pontificia Universidad Javeriana. Facultad de Ciencias Políticas y Relaciones Internacionales. Recuperado de https://www.academia.edu/35380923/El_Estudio_de_Caso_simple_un_dise\%C3 \%B10_de_investigaci\%C3\%B3n_cualitativa

Álvarez-Gayou, J.L. (2007). Cómo hacer investigación cualitativa. Fundamentos y metodología. Paidos

Andrews, F. M. y Withey, S. B. (1976). Social indicators of well-being: American's Perceptions of life quality. Penum Press.

https://deepblue.lib.umich.edu/bitstream/handle/2027.42/43678/11205_2004_Artic le_BF00303860.pdf?sequence $=1$

Ardila, R. (2003). Calidad de vida: una visión integradora. Revista Latinoamericana de Psicología, 35(2), 161-164. https://www.redalyc.org/pdf/805/80535203.pdf

Aristóteles. (2007). Ética nicomáquea. (R.A. Gómez, Trad.). UNAM. (original publicado en c. 384-322 a.C.).

Arita, B. (2016). Políticas de bienestar: indicadores objetivos y subjetivos para la medición de la calidad de vida en Sinaloa, México. En D. Gomez-Alvrez. y O. V. Ortiz (Comp), El bienestar subjetivo en América Latina (pp. 75-87). Universidad de Guadalajara. 
Baxter, J. y Eyles, J. (1997). Evaluating Qualitative Research in Social Geography: Establishing "Rigor" in Interview analysis. Transactions of the Institute of British Geographers, 22(4) 5005-525. doi:10.1111/j.0020-2754.1997. 00505.x

Bedregal, P., Besoain, C., Reinoso, A. y Zubarew, T. (2017). La investigación cualitativa: un aporte para mejorar los servicios de salud. Revista médica de Chile, 145(3), 373379. http://www.revistamedicadechile.cl/ojs/index.php/rmedica/article/view/5218

Bobes, J., González, P., Bousoño, M., y Suárez R. E. (1993). Desarrollo histórico del concepto de calidad de vid. Psiquiatría, 5(6), 5-9.

https://www.unioviedo.es/psiquiatria/wpcontent/uploads/2017/03/1993_Bobes_Desarrollo.pdf

Calderón, C. (2002). Criterios de calidad en la Investigación Cualitativa en Salud (ICS): Apuntes para un debate necesario. Revista Española de Salud Pública, 76(5), 473-482. http://scielo.isciii.es/scielo.php?script=sci_arttext\&pid=S1135$57272002000500009 \& \operatorname{lng}=$ es\&tIng=es.

Campbell, A., Converse, P.E. y Rodgers, W. L. (Ed.) (1976). General Sense of Well-Being. The quality of American life: Perceptions, evaluations and satisfactions. Editorial Russell Sage Foundation. Recuperado de https://bit.ly/37bAEcV

Carpio, C., Pacheco, V., Flores, C., y Canales, C. (2000). Calidad de vida: un análisis de su dimensión psicológica. Revista Sonorense de Psicología, 14(1 y 2), 3-15. https://bit.ly/3IRNmlb

Casas, F. (1999). Calidad de vida y calidad humana. Papeles del Psicólogo,74. de http://www.papelesdelpsicologo.es/resumen?pii=812

Casas, F. (2015). Bienestar material y bienestar subjetivo. Universitat de Barcelona. https://www.educo.org/Educo/media/Documentos/Estudios/Bienestar-vol4ferran.pdf

Coleman, J. (1988). Social Capital in the Creation of Human Capital. American Journal of Sociology, sup., $94 \mathrm{~S}$.

https://www.econ.msu.ru/cmt2/lib/c/477/File/Social\%20Capital\%20in\%20the\%20C reation\%20of\%20Human\%20Capital.pdf

Conill, G., P. (1984). Geografía para tiempos difíciles. Escenarios Latinoamericanos de la Calidad de Vida. Revista Nueva Sociedad, 75, 41-48. https://nuso.org/media/articles/downloads/1227_1.pdf

Daymon, Ch., y Holloway, I. (2010). Qualitative research methods in public relations and marketing communication. Routledge. 
Dennis, R.E., Williams, W., Giangreco, M.F., y Cloninger, CH.J. (1993). Quality of Life as Context for Planning and Evaluation of for Services for People with Disabilities. Excepcional Children, 6(59), 499-512.

https://www.uvm.edu/sites/default/files/Center-on-Disability-and-CommunityInclusion/GiangrecoEC93596499-512.pdf

Diener, E., Seligman, M., Choi, H., y Oishi, S. (2018). Happiest people revisited. Perspective on Psychological Science, 13(2) 176-184. doi:10.1177/1745691617697077

Evans, D. R., Burns, J. E., Robinson, W. E., y Garrett, O. J. (1985). The quality of, life questionnaire: A multidimensional measure. American Journal of Community Psychology, 13, 305-322. doi:10.1007/BF00914935

Fernández-López, J. A., Fernández-Fidalgo, M., y Cieza, A. (2010). Los conceptos de calidad de vida, salud y bienestar analizados desde la perspectiva de la Clasificación Internacional del Funcionamiento (CIF). Revista Española de Salud Pública, 84(2), 169-184.

http://scielo.isciii.es/scielo.php?script=sci_arttext\&pid=S1135$57272010000200005 \& \operatorname{lng}=$ es\&tlng=es.

Ferris, A. (2006). A theory of social structure and the quality of life. Applied Research in Quality of Life, 1, 117-123.

Flick, U. (2012). Introducción a la investigación cualitativa. Morata.

Gordziejczuk, M.A. y Mikkelsen, C.A. (2019). Reflexiones sobre calidad de vida y espacio geográfico en Argentina: aportes al estado del arte. Estudios Socioterritoriales. Revista Geografía, (27). https://doi.org/10.37838/unicen/est.27-052

Gomez-Alvrez, D. y Ortiz, O., V. (Comp). (2016). El bienestar subjetivo en América Latina. Universidad de Guadalajara.

González, P., Díaz, J., Rodríguez, L.F. y Bobbes, J. (1993). Modelos de Calidad de Vida. Revista Psiquiatría. (V). 6. P. 18-22. https://www.unioviedo.es/psiquiatria/wpcontent/uploads/2017/03/1993_Gonzalez_Modelos.pdf

González, B.F., Escoto, P.L. y Chávez, L. J. (2017). Estadística aplicada en Psicología y Ciencias de la salud. Manual Moderno.

Grinnell, R. (1997). Social work research \& evaluation: Quantitative and qualitative approaches. Peacock Publishers.

Hernández, A. (1998). Amartya Sen: Ética y Economía. La ruptura con el bienestarismo y la defensa de un consecuencialismo amplio y pluralista. Cuadernos de Economía, 
XVII(29), 137-162.

https://revistas.unal.edu.co/index.php/ceconomia/article/view/11527

Ibáñez, T. e Iñiguez, L. (1996). Aspectos metodológicos de la Psicología Social Aplicada. En J.L. Álvaro, A. Garrido, J.R. Torregrosa (Coord.). Psicología Social Aplicada. (pp. 57-82) McGraw-Hill.

Iñiguez, L. (2004). El debate sobre metodología cualitativa versus cuantitativa. En Curso de investigación cualitativa: fundamentos, técnicas y métodos. Recuperado de https://larrycamacho.jimdofree.com/app/download/10003455670/Debate+cualitativ a+cuantitativa+LUPICINIO.pdf?t=1571022226

Izcara, P., S. P. (2014). Manual de la Investigación Cualitativa. Colección Argumentos. Fontamara

Jorgensen, D.L. (1989). Participant Observation: A Methodology for Human Studies. Sage Land, K.C. y Michalos, A. C. (2018). Fifty Years After the Social Indicators Movement: Has the Promise Been Fulfilled? An Assessment an Agenda for the Future. Social Indicators Research, (135), 835-868. https://doi.org/10.1007/s11205-017-1571-y

Latiesa, M. (1994). Validez y fiabilidad de las observaciones sociológicas. En M. A. García Ferrando, J. Ibáñez y F. Alvira (Comps.), El análisis de la realidad social. Métodos y técnicas de investigación (pp. 335-364). Alianza Universidad Textos.

Lecomte, J. (2018). ¡El mundo va mucho mejor de lo que piensas! Recuperado de https://cutt.ly/YvHjncb

Lichtman, M. (2013). Qualitative Research for social science. Sage Publications.

López-López, A. (2015). Young people's rural quality of life in the Colombian Andes: a qualitative study using triangulation. En G. Tonon (Ed.), Qualitative studies in quality of life. Methodology and Practice (pp. 179-216). Social Indicators Series Springer.

Lucero, P. I. (2009). La construcción social del espacio geográfico: Reflexiones metodológicas. En G. Tonon (Comp.), Reflexiones Latinoamericanas sobre Investigación Cualitativa (pp. 124-145). Prometeo.

Lutz, B. (2014). Formación histórica de la sociología rural: proceso de civilización del indio y del campesino en México (1870-1960). Sociológica, 29(81) 161-197 http://www.scielo.org.mx/scielo.php?script=sci_arttext\&pid=S018701732014000100005

Lyn, R. y Morse, J. (2012). Readme first for a user guide to qualitative methods. Sage Publications. 
Marshall, C. y Rossman, G. B. (1999). Designing Qualitative Research. SAGE.

Martínez Miguélez, M. (2006). Validez y confiabilidad en la metodología cualitativa. Paradígma, 27(2), 7-33.

Maslow, A.H. (1943). A theory of human motivation. Psychological Review, 50(4), 370-396. http://dx.doi.org/10.1037/h0054346

Max-Neef, M. (1994). Desarrollo y necesidades humanas. Desarrollo a Escala Humana. Recuperado de http://www.daghammarskjold.se/wpcontent/uploads/1986/08/86_especial.pdf

Michalos, A. C. (1980). North American Social Report: A comparative Study of the Quality of Life in Canada and USA from 1964 to 1974. Reidel Publishing Company.

Mikkelsen, C.A. (2007). Ampliando el estudio de la calidad de vida hacia el espacio rural. El caso del partido del general Pueyrredón. Argentina. Revista Hologramática, 6(4) $52-48$.

Monje, A. C.A. (2011). Metodología de la investigación Cualitativa. Guía didáctica. Recuperado de https://www.uv.mx/rmipe/files/2017/02/Guia-didacticametodologia-de-la-investigacion.pdf

Moreno Jiménez, B y Ximénez Gómez, C. (1996). Evaluación de la calidad de vida. En G. Buela Casal, et al. (Eds.), Manual de evaluación en psicología clínica y de la Salud (pp. 1-94). Siglo XXI. Recuperado de https://www.infogerontologia.com/documents/burnout/articulos_uam/calidaddevid a.pdf

Noll, H-H. (2002). Towards a European System of Social Indicators: Theoretical Framework and System Architecture. Social Indicators Research, 58(1-3), 47-87. doi.org/10.1023/A:1015775631413

Patton, M. Q. (1990). Qualitative Evaluation and Research Methods. Sage Publications.

Peccei, A. (1976). La qualità humana. Milano: Arnoldo Mondadori. (Trad. A. Mondadori). En Informes al club de Roma. Taurus.

Platón. (2000). La República. (A. Gómez Robledo, trad.). UNAM. (original publicado en c. 428-348 a.C.)

Punch, K. F., y Oancea, A. (2014). Introduction to Research Methods in Education. Sage Publications.

Putnam, R., R. Leonardi, R. y Nanetti, R. (1993). Making Democracy Work: Civic Traditions in Modern Italy, Princeton University Press. Recuperado de https://cutt.ly/avHjITv 
Quecedo, R. y Castaño, C. (2002). Introducción a la metodología de investigación cualitativa Revista de Psicodidáctica, 14, 5-39.

https://www.redalyc.org/pdf/175/17501402.pdf

Rojas, M. (2014). El estudio científico de la felicidad. Fondo de Cultura Económica.

Recuperado de

http://www.fondodeculturaeconomica.com/subdirectorios_site/libros_electronicos/ desde_la_imprenta/014582R/files/publication.pdf

Seale, C. (2001). Qualitative methods: Validity and reliability. European Journal of Cancer Care, 10(2), 134-135. doi:10.1046/j.1365-2354.2001. 0253b.x

Silva, J., Keulenaer, F. y Johnstone, N. (2012). Environmental Quality and Life Satisfaction: Evidence Based on Micro-Data. (OECD Environment Working Papers No.44). Recuperado de https://www.oecd-ilibrary.org/docserver/5k9cw678dlr0en. pdf?expires $=1614127578 \&$ id $=$ id \&accname $=$ guest $\&$ checksum $=4$ CEBC6F5C 10 A4C73C164DDF602DD6215

Sirgy, M.J. (2012). The Psychology of Quality of Life. Hedonic Well-Being, Life Satisfaction and Eudaimonia. (2a ed.). Springer. doi: 10.1007/978-94-007-4405-9

Sócrates. (2006). Sócrates. En Fernández Labastida, y Mercado, J.A. (Eds). Philosophica: Enciclopedia filosófica on line. Recuperado de http://www.philosophica.info/voces/socrates/Socrates.html

Taylor, S.J. y Bogdan, R. (1987). Introducción a métodos cualitativos de investigación. La búsqueda de significados. Paidós.

Tonon, G. (2003). Calidad de vida y desgaste profesional: una mirada del síndrome de burnout. Espacio Editorial.

Tonon, G. (2005). Un lugar en el mundo: la universidad como espacio de integración social para los estudiantes. Revista Hologramática. (3) 41-49. www.cienciared.com.ar/ra/doc.php?n=134

Tonon, G. (2008). Desigualdades sociales y oportunidades ciudadanas. Espacio Editorial.

Tonon, G. (2009a). Calidad de vida laboral de profesionales de la salud. Vertex Revista Argentina de Psiquiatría, $X X(88), 411-417$.

Tonon, G. (comp.) (2009b). Reflexiones Latinoamericanas sobre Investigación Cualitativa. Prometeo.

Tonon, G. (Ed.) (2015). Qualitative Studies in Quality of Life. Methodology and practice. Springer. 
Toscano, W.N. y Molgaray, D. (2019). The Research Studies on Quality of life in South America. Applied Research in Quality of Life, 14, 573-588. doi:10.1007/s11482018-9605-4

Urzúa, A. y Caqueo-Urízar. (2012). Calidad de Vida: Una revisión teórica del concepto. Terapia Psicológica, 30(1), 61-71. https://scielo.conicyt.cl/pdf/terpsicol/v30n1/art06.pdf

Veenhoven, R. (2000). Well-being in the welfare state: Level not higher, distribution not more equitable. Journal of Comparative Policy Analysis: Research and Practice, 2(1), 91-125. doi:10.1080/13876980008412637

Vieytes, R. (2009). Campos de aplicación y decisiones de diseño en la investigación cualitativa. En A. Merlino (Coord.), Investigación cualitativa en ciencias sociales. Temas, problemas y aplicaciones (pp. 43-84). Cengage Learning.

Villegas, M. M. y González, F. E. (2011). La investigación cualitativa de la vida cotidiana, medio para la construcción de conocimiento sobre lo social a partir de lo individual. Psicoperspectivas. Individuo y Sociedad, 10(2),147-175. https://www.psicoperspectivas.cl/index.php/psicoperspectivas/article/view/147/175

Wynn, P. y Money, A. (2009). Qualitative Research and Occupational Medicine. Occupational Medicine, (59), 138-139.

\section{Otros documentos consultados}

CONEVAL. (2017). Medición de la pobreza, resultados a nivel nacional y por entidad federativa 2010-2014. Recuperado de http://www.coneval.org.mx/Medicion/MP/Paginas/Pobreza_2014.aspx

OMS, (1994). Quality of Life Assessment: An annotated bibliography. Recuperado de https://apps.who.int/iris/bitstream/handle/10665/61629/WHO_MNH_PSF_94.1.pdf

PNUD (1990). Informe de Desarrollo Humano 1990. Recuperado de http://hdr.undp.org/sites/default/files/hdr_1990_es_completo_nostats.pdf 